American Journal of Bioinformatics 1 (1): 64-69, 2012

ISSN 1948-9862

(C) 2012 Science Publications

\title{
An Optimal Approach for Medical Image Analysis
}

\author{
J. Umamaheswari and G. Radhamani \\ Department of Computer Science, \\ School of Information Technology and Science, \\ GRD College of Science, Affiliated to Bharathiyar University, Tamilnadu, India
}

\begin{abstract}
Problem statement: Image segmentation is the process that subdivides an image into its constituent parts and extracts the objects. It is one of the most critical tasks in automatic image analysis because the subdivided results will affect all the subsequent processes of image analysis, such as object representation and description, feature measurement and even the following higher level tasks such as object classification and scene interpretation by optimized results. Approach: In this study, we proposed an optimal approach for medical image segmentation based on the combination of Particle Swarm Optimization (PSO) and Global Minimization by Active Contour (GMAC) methods. PSO is a population based new evolutionary algorithm in the field of image segmentation where the image homogeneous part can be detected. The grouped part from PSO is again treated with GMAC to reduce the complex region of image parts. Results: The feasibility of these algorithms for analyzing is presented through experimental investigation. The simulation results give that the proposed optimal approach gives efficient results for image segmentation. Conclusion/Recommendation: The performance of the proposed study is compared with the existing traditional algorithm and real time medical diagnosis image.
\end{abstract}

Key words: Particle Swarm Optimization (PSO), Global Minimization by Active Contour (GMAC), Parametric Active Contour (PAC), Swarm Intelligence (SI), Geometric Active Contour (GAC), existing traditional algorithm

\section{INTRODUCTION}

Segmentation is one of the most important techniques for image processing $\mathrm{Hu}$ et al. (2009). The purpose of segmentation is to partition an image into distinct, semantically meaningful entities by defining boundaries between features and objects in an image based on some constraint, or homogeneity predicate. Image segmentation methods fall into six categories: Pixel based segmentation Ruz et al. (2005), Level set segmentation, Region based segmentation Jayadevappa et al. (2009), Edge based segmentation by Bresson et al. (2007), Edge and Region Hybrid segmentation Chuang et al. (2006) and Clustering based segmentation Airouche et al. (2009); Mezhoud and Hachouf (2011); Bonabeau et al. (1999) and Eberhart et al. (2001).

For optimal approach, a lot of techniques are available to minimize a convex cost function. When PSO or GMAC techniques are processed individually to estimate the cost function which is a nonlinear function, it is very difficult to statistically determine the function which can be used as a minimizer for the given function. In our study, the optimal segmentation is processed to meet the cost function by the combination of PSO and GMAC.
Need for medical image segmentation: Medical image segmentation is a difficult problem due to the fact that medical images commonly have poor contrast and missing details due to different types of noise. The segmentation methods depend on modality and dimension of imaging because of the high dependency of factors like disease type and image features. Likewise, segmentation needs the image interpretation because of its dependency on the considered applications.

Particle Swarm Optimization (PSO): PSO based on the study of Bird flocks searching for food is used for optimal segmentation by Allili et al. (2004) and Airouche et al. (2009). In the active contour model, the segmentation of an image plays a very important role. Here, different initial conditions in the evolution will give different segmented region, where the results will not be satisfactory using (Lee and Park, 2006) Ginneken et al. (2002), Kharrat et al., 2011).

To improve the minimization of active contour method GMAC is used for contour based level set segmentation.

Corresponding Author: Umamaheswari, J., Department of Computer Science, School of Information Technology and Science, GRD College of Science, Affiliated to Bharathiyar University, Tamilnadu, India 
Global Minimization by Active Contour Method (GMAC): GMAC based segmentation was used for simplifying the image. The optimization techniques are based on biologically inspired techniques, after studying the functioning of various organisms like ants, particle swarms, bird flocks and even the evolution of human population. Each of these gives rise to a different technique, which are heavily used in these days in day to day optimization problems. A few algorithms were proposed to determine the global minimization by Meng et al. (2007) and Bresson et al. (2007).

\section{MATERIALS AND METHODS}

A framework for the optimal methodology: This study presents the optimal approach on the combination of PSO and GMAC algorithm. The detailed explanation of the detection system is described in Fig. 1 which shows an optimal segmentation system. The preprocessing is the first stage which starts the process, produce noise suppression and image enhancement. The second process consists of PSO with GMAC segmentation for irregular brain detection. The performance of the segmentation is compared with other segmentation method. The optimal image detection system framework is shown in Fig. 1.

The aim is to produce the optimal segmented regions within few seconds, which is based on the following methods:

- Particle Swarm Optimization (PSO)

- Improved Global Minimization by Active Contour (GMAC) with PSO

Particle Swarm Optimization (PSO): Recently, Swarm Intelligence (SI) has been applied in numerous fields including optimization. One of SI methods performing well in solving optimization problems is Particle Swarm Optimization (PSO). PSO is a stochastic search method that was developed in 1995 based on the sociological behavior of bird flocking. The algorithm of PSO is easy to implement and has been successfully applied to solve a wide range of optimization problems in many fields such as image processing fields including image segmentation. Image segmentation is a low-level image processing task aiming at partitioning an image into homogeneous regions. The segmenting curve by PSO is defined in the following Eq. 1:

$$
\Phi(\mathrm{x}, \mathrm{y})=\bar{\Phi}(\mathrm{x}, \mathrm{y})+\sum_{\mathrm{i}=1}^{\mathrm{k}}(\mathrm{x}, \mathrm{y}) \mathrm{w}_{\mathrm{i}} \Phi_{\mathrm{i}}
$$

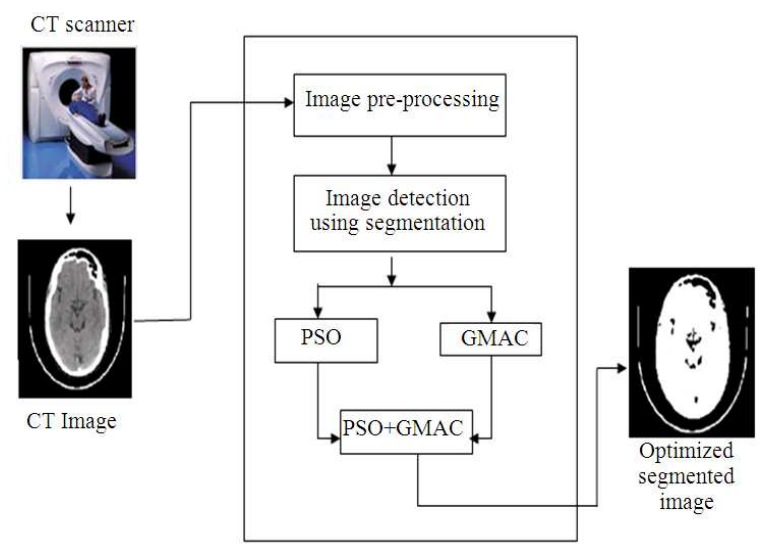

Fig. 1: Optimized segmentation using PSO and GMAC

where, $\mathrm{k}$ is the number of principle eigenshapes, $\mathrm{w}_{\mathrm{i}}, \mathrm{I}$ $=1,2, \ldots, \mathrm{k}$ are the weights for these eigenshapes and these weights are ranged from $-\sigma_{i}$ to $\sigma^{2}{ }_{i}$ (where $\sigma_{i}$ are the eigenvalues corresponding with these ${ }^{\text {th }} i$ eigenshape). In addition, we consider the pose parameters; $\mathrm{a}, \mathrm{b}$ for translation, $\mathrm{h}$ for scaling and $\theta$ for the rotation angle, which incorporated into this framework using an affine transform. Therefore each particle $\mathrm{P}$ in the $\mathrm{PSO}$ population is represented as $\mathrm{P}=$ $\left[\left(w_{i}, I=1,2, \ldots, k\right), a, b, h, \theta\right]$ and it represents a segmenting curve.

The fitness of each particle in this study represents how the corresponding curve segments the image. So in the proposed technique, we tend to maximize the fitness function proposed in (Allili et al., 2004; Airouche et al., 2009). This fitness Function (FT) is formulated as Eq. 2:

$\mathrm{FT}=600\left(\mathrm{~A}+\left(1 \_\mathrm{B}\right)\right)$

where, $\mathrm{A}$ is the fraction of pixels inside the segmenting curve that are labelled "true" and B is the fraction of the pixels outside the segmenting curve that are labelled "true". The maximization of this fitness function means that more desired pixels are gathered inside the segmenting curve.

The PSO algorithm implementation: The PSO image segmentation is carried out by adjusting the curve parameters according to the undesirable object, the segmentation process is done by the following sequence:

Select the curve parameters arbitrarily from the range specified in Table 1 and 2 and create the Corresponding level set functions

- $\quad$ Segment the image by using the curves derived from the generated level set functions 
Table 1: PSO algorithm configuration

\begin{tabular}{lr}
\hline Swarm size (the no. of segmenting curves) & \multicolumn{1}{c}{25} \\
\hline Max. no. of epochs & 100.0 \\
Local best influence & 2.0 \\
Global best influence & 2.0 \\
Initial inertia weight & 0.9 \\
Final inertia weight & 0.4 \\
Epoch when inertia weight at final value & 80.0 \\
\hline
\end{tabular}

Table 2: Segmenting curve configuration

\begin{tabular}{lll}
\hline Parameter name & Parameter range & Maximum velocity \\
\hline wi $, \mathrm{i}=1,2, \ldots, \mathrm{k}$ & $-\sigma_{\mathrm{I}}-\sigma_{\mathrm{i}}$ & $\frac{\sigma_{\mathrm{i}}}{5}$ \\
& & 0.5 \\
$\mathrm{a}, \mathrm{b}$ & $-20 \sim 20$ & 0.5 \\
$\mathrm{~h}$ & $0.5 \sim 2$ & 12 \\
$\theta$ & $-90 \sim 90$ & \\
\hline
\end{tabular}

- Measure the fitness of each curve by computing the fitness function described in the PSO algorithm

- and determine the best curve

- Update the curve parameters according to the PSO algorithm equations.

- Create the level set functions of the new parameters and repeat Step-2

- Repeat Step-3

- If the best curve is not changed for more than 10 epochs, produce the segmentation results; else go to Step-4

The PSO and segmenting curve configuration is shown in Table 1 and 2.

\section{Disadvantages of PSO-based approaches:}

- Lacking somewhat of a solid mathematical foundation for analysis

- Some limitations in real-time applications, such as in the $5 \mathrm{~min}$ dispatch with network constraints Due to relatively longer computation time (Possibility for the off-line real-world problems such as in the Day-ahead electricity markets)

- Still having the problems of dependency on initial conditions, parameter values, difficulty in finding the optimal design parameters, stochastic characteristics of the final outputs

- The major drawback of PSO, like in other heuristic optimization techniques, is that it lacks somewhat a solid mathematical foundation for analysis. The PSO is a variant of stochastic optimization techniques requiring relatively a longer computation time than mathematical approaches

- It still has the problems of dependency on initial point and parameters, difficulty in finding their optimal design parameters and the stochastic characteristic of the final outputs
The Segmented results produced by the PSO algorithm consist of some irregular regions and consequently to improve the results the GMAC level set method is used for segmenting curve. The following explains the GMAC method to produce the optimal output.

Improved GMAC with PSO: The idea of segmentation based on active contour models work efficiently. A contour deforms until it reaches the boundary of the object to be detected. This is accomplished by constructing and solving a Partial Differential Equation (PDE) that directs the evolution of the contour from its initial position and shape. There are mainly two kinds of active contour, namely, Parametric Active Contour (PAC) and Geometric Active Contour (GAC). PAC is based on energy minimization, whereas GAC is based on the theory of curve evolution and geometric flow. In the case of the GAC model, the existence of local minima can prevent the segmentation of significant objects lying in images. An image segmentation model provides independently the correct result considering the initial condition, which means that a global minimum of a convex function. New active contour energies based on the GAC model and the values of global minimum region corresponds with the expected segmentation result has been used. Even though the noisy input is given to GMAC the improved method removes the noise while preserving the edges in the image for segmentation.

In the active contour model, the segmentation of an image plays a very important role. Here, different initial conditions in the evolution will give different segmented region, where the results will not be satisfactory. This unsatisfactory result is the minimization problem of the active contour.

A few algorithms were proposed in Ruz et al. (2005) to determine the global minimization which is to modify the Rudin, Osher and Fatemi (ROF) (Bresson et $a l ., 2007)$ energy given below Eq. 3:

$\mathrm{E}_{\mathrm{ROF}}(\mathrm{v}, \lambda)=\int_{\Omega}|\nabla v|+\frac{\lambda}{2} \int_{\Omega}|\mathrm{v}-\mathrm{f}|^{2}$

By first replacing the Total Variation (TV) by a weighted TV and then, more importantly, changing the measure in the fidelity term from the square of the $\mathrm{L}_{2}$ to the $\mathrm{L}_{1}$. This gives Eq. 4:

$\mathrm{E}_{2}(\mathrm{v}, \lambda)=\int_{\Omega}^{\mathrm{c}} \mathrm{g}(\mathrm{f})|\nabla v|+\lambda \int_{\Omega}^{\mathrm{c}}|\mathrm{v}-\mathrm{f}|^{2}$

where, $g(f)=1 /\left(1+\beta|\nabla f|^{c}\right)$ 
As in the study (Sonka et al., 1999), the characteristic function of a set with boundary given by the curve $C$ the minimizer of the above energy $E_{2}$ is the same as the minimizer of the active contour energy Eq. 5:

$$
E_{2 C}=\int_{c} g(f) d s
$$

With $\mathrm{f}$ approximated by a binary function of a region $2_{C}$. Numerically, the minimization problem is convex. The global minimization active contour method avoids the uncertainty disturbances. To improve the segmentation results with high homogeneity the active contour method called GMAC is used. The results produced by PSO are again processed by the Fast GMAC algorithm for minimizing the segmenting region. The result produced by the optimal approach gives the less computation time and low minimal error. The Global minimal energy level is obtained by the following equation Eq. 6:

$$
E_{2 C}=\int g(f) d s
$$

The GMAC will reduce the noise and fast minimization of the region take place with better homogeneity. The advantage of GMAC is to improve the result with fast minimization.

Hence in our approach the better results are obtained by the following formation of the equation Eq. 7:

$$
\mathrm{E}_{2 \mathrm{C}}=\int \Phi(\mathrm{x}, \mathrm{y}) \overline{\mathrm{ds}}_{\mathrm{c}}
$$

where, the $\mathrm{E}_{2 \mathrm{C}}$ is the global minimal energy level for segmenting curve $\mathrm{C}$ (region). The $\bar{\Phi}(\mathrm{x}, \mathrm{y})$ is the PSO level set method for segmenting curve.

\section{RESULTS AND DISCUSSION}

The experiments are presented to demonstrate the practice and the performance of the proposed novel image segmentation approach. Moreover, Otsu's (clustering based) method (Airouche et al., 2009) PSO method, Level set method by senthilkumar (Bresson et al., 2007) and Fast GMAC method (Bonabeau et al., 1999 ) is carried out for comparison. Both real images are $256 \times 256$ in size. The first original image with Otsu and FCMT results are shown in Fig. 2a-c. Figure $2 \mathrm{~d}-\mathrm{g}$ illustrate the segmented images using GMAC method and PSO method.

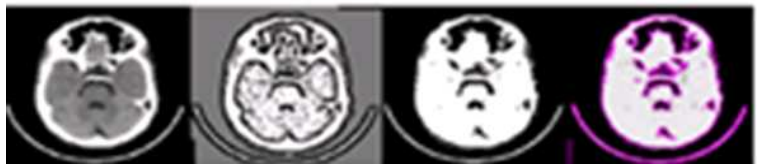

(a)

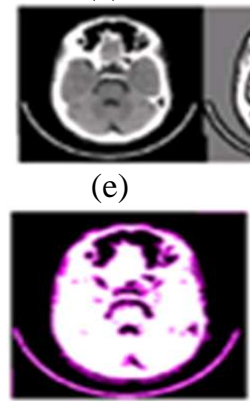

(i) (b)

(c)

(d)

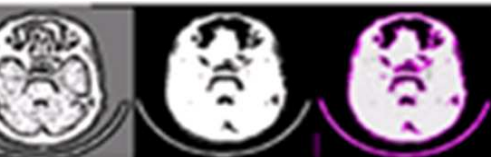

(f)

(g)

(h)

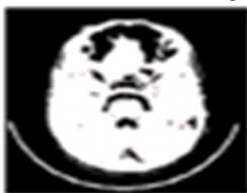

(j)

(k)
Fig. 2: Image results of Different Segmentation Algorithm, (a) Original Image, (b) Otsu Method, (c) FCMT method, (d) to (e) GMAC Method, (f) to (h) PSO Method (i) to (k) PSO+GMAC Optimal approach

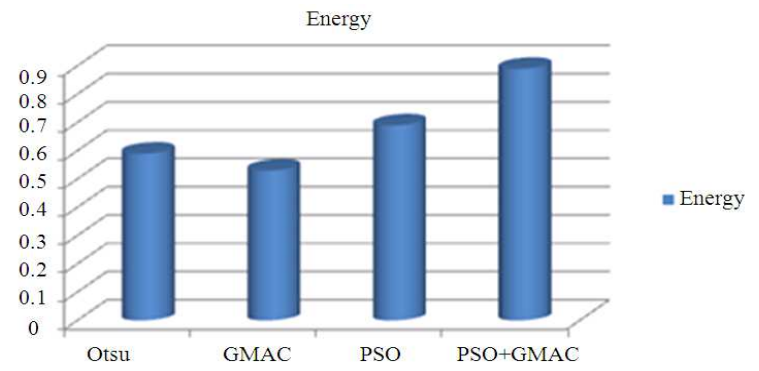

Fig. 3: Energy displays for different segmentation method

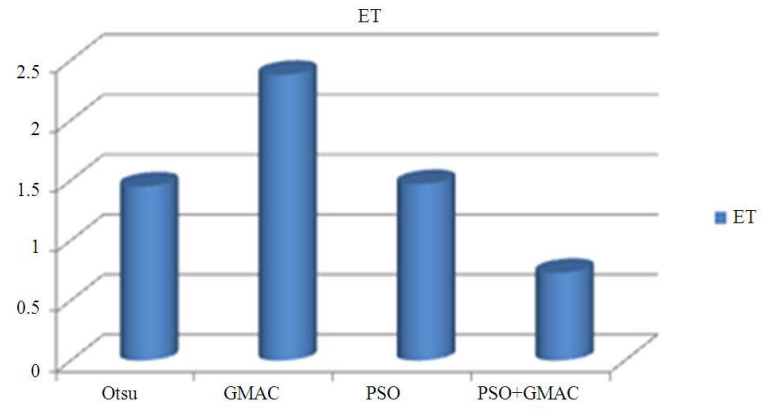

Fig. 4: ET display for different segmentation method

Figure $2 \mathrm{i}$ shows the graph of $\mathrm{g}$ best in PSO. Figure $2 \mathrm{j}-\mathrm{m}$ represents the proposed approach, respectively. As contrasted with GMAC and PSO results, the regions are extracted completely by the proposed approach. 
Table 3: Parametric Evaluation of different Segmentation method

\begin{tabular}{llll}
\hline Method & Energy & Evaluation(sec) & Time \\
\hline Otsu & 0.59 & 1.45 & \\
GMAC & 0.53 & 2.38 & \\
PSO & 0.69 & 1.47 & \\
PSO with GMAC & 0.89 & 0.73 & \\
\hline
\end{tabular}

In the proposed optimal approach even the minute region is taken into consideration which gets minimized to produce the segmented results. Figure 3 and 4 gives the energy and ET values for different segmentation algorithms.

The energy value and evaluation time is calculated based on the following conditions, the gray level energy shows how the gray levels are distributed. It is formulated as:

$$
\mathrm{E}(\mathrm{x})=\sum_{\mathrm{i}=1}^{\mathrm{x}} \mathrm{p}(\mathrm{x})
$$

where, E (x) represents the gray level energy with 256 bins and $\mathrm{p}$ (i) refers to the probability distribution functions, which contains the histogram counts. The energy reaches its maximum value of 1 when an image has a constant gray level. The larger energy value corresponds to the lower number of gray levels, which means simple. The smaller energy corresponds to the higher number of gray levels, which means complex. The following Table 3 shows the different parametric evaluation for segmentation algorithm. The energy value is high for a better quality image.

Evaluation Time (ET) depends essentially on the computing system clock time-period, yet it is not necessarily dependent on the clock time alone. Rather, in addition to the clock-period, it depends on the memorysize, the input data size and the memory access time. However, the measure ET is very important in case of real-time application. The evaluation time is minimized when they are jointly operated.

The result of Otsu method is very sensitive to object's noise. As contrasted with PSO and Levelset results, the boundary is properly separated by the proposed approach. It is very difficult to evaluate segmentation results and to compare the related methods. However, based on the following criteria (1) segmented regions should be uniform and homogeneous, (2) region interiors should be simple, (3) adjacent regions should be significantly different and (4) boundaries of each region should be simple and spatially accurate (Sharma and Aggarwal, 2010). The optimal approach proves efficient based on Energy and ET values.

\section{CONCLUSION}

In case of medical image segmentation the aim is to study anatomical structure, identify the region of interest, measure abnormality and help doctors in planning for early diagnosis. In this study, we proposed an optimal approach for medical image segmentation based on the combination of Particle Swarm Optimization (PSO) and Global Minimization by Active Contour (GMAC) methods. The PSO is a population based new evolutionary algorithm in the field of image segmentation where the image homogeneous part can be detected. The grouped part from PSO is again treated with GMAC to reduce the complex region of image parts. The simulation result gives that the proposed optimal approach gives efficient results for medical image segmentation based on parametric metrics.

Future scope: Our approach is robust when compared to other methods. It still consists of some problem in selecting the parameter configuration. In our future study, we will investigate better and more efficient ways to solve the computational problems. Our goal is to achieve real time interactive image segmentation of arbitrary number of classes using the optimization framework with less computational time.

\section{REFERENCES}

Airouche, M., L. Bentabet and M. Zelmat, 2009. Image segmentation using active contour model and level set method applied to detect oil spills. Proceedings of the World Congress on Engineering, July 1-3, London, U.K., pp: 1-5.

Allili, M.S., D. Ziou and L. Bentabet, 2004. A robust level set approach for image segmentation and statistical modelling. Proceedings of Advanced Concepts for Intelligent Vision Systems, Aug. 31Sept. 3, Brussels, Belgium, pp: 243-251.

Bonabeau, E., M. Dorigo and G. Theraulaz, 1999. Swarm Intelligence: From Natural to Artificial Systems. 1st Edn., Oxford University Press, Oxford, ISBN-10: 0195131592, pp: 307.

Bresson, X., S. Esedoglu, P. Vandergheynst, J.P. Thiran and S. Osher, 2007. Fast global minimization of the active contour/snake model. J. Math. Imag. Vision, 28: 151-167.

Ginneken, B.V., A.F. Frangi, J.J. Staal, B.M.H. Romeny and M.A. Viergever, 2002. Active shape model segmentation with optimal features. IEEE Trans. Med. Imag., 21: 924-933. DOI: 10.1109/TMI.2002.803121 
Hu, Grossberg and Mageras, 2009. Survey of recent volumetric medical image segmentation techniques. Biomed. Eng. DOI: 10.5772/7865

Jayadevappa, D., S.S. Kumar and D.S. Murty, 2009. A hybrid segmentation model based on watershed and gradient vector flow for the detection of brain tumor. Int. J. Signal Process. Image Process. Patt. Recogn., 2: 29-42.

Chuang, K.S., H.L. Tzeng, S. Chen, J. Wu and T.J. Chen, 2006. Tzong-Jer, 2006. Fuzzy c-means clustering with spatial information for image segmentation. Comput. Med. Imag. Graphics, 30: 9-15.

Eberhart, R.C., Y. Shi and J. Kennedy, 2001. Swarm Intelligence. 1st Edn., Elsevier, Burlington, ISBN10: 0080518265 , pp: 512.

Kharrat, A., K. Gasmi and M. Abid, 2011. Medical image classification using an optimal feature extraction algorithm and a supervised classifier technique. Int. J. Software Sci. Comput. Intell., 3: 1-15.

Lee, K.Y. and J.B. Park, 2006. Application of particle swarm optimization to economic dispatch problem: Advantages and disadvantages. Proceeding of the IEEE Power System Conference and Explosion, Oct. 29-Nov. 1 2006, IEEE Xplore Press, Atlanta, GA, pp: 188-192. DOI: 10.1109/PSCE.2006.296295
Meng, Y., O. Kazeem and J.C. Muller, 2007. A hybrid ACO/PSO control algorithm for distributed swarm robots. Proceedings of the IEEE Swarm Intelligence Symposium, Apr. 1-5, IEEE Xplore Press, Honolulu, HI, pp: 273-280. DOI: 10.1109/SIS.2007.367948

Mezhoud, N. and F. Hachouf, 2011. A new hybrid method for medical image segmentation. J. Theoritical Applied Inform. Technol., 26: 1-15.

Ruz, G.A., P.A. Estevez and C.A. Perez, 2005. A neurofuzzy color Image segmentation method for wood surface defect detection. Forest Prod. J., 55: 52-58.

Sharma, N. and L.M. Aggarwal, 2010. Automated medical image segmentation techniques. J. Med. Phys., 35: 3-14. PMID: 20177565

Sonka, M., V. Hlavac and R. Boyle, 1999. Image Processing, Analysis and Machine Vision. 2 Edn., PWS Publication, ISBN-10: 053495393X, pp: 770. 